\title{
Fusion of Uncertain Location Data From Heterogeneous Sources
}

\author{
[Invited Keynote]
}

\author{
Goce Trajcevski \\ Dept. of EECS \\ Northwestern University \\ Evanston, IL, USA \\ goce@eecs.northwestern.edu
}

\section{Categories and Subject Descriptors \\ H.4 [Information Systems Applications]: Miscellaneous}

\section{General Terms}

Fusion, Uncertainty, Heterogeneity

\section{INTRODUCTION AND MOTIVATION}

Many applications of high societal relevance - e.g., transportation and traffic management, disaster remediation, location-aware social networking, (tourist) recommendation systems, military logistics (to name but a few) - rely on some kind of Location Based Services (LBS). The crucial components to support such services, in turn, rely on efficient techniques for managing the data capturing the information pertaining to the whereabouts in time of the moving entities - storing, retrieving and querying such data. Traditionally, such topics were subjects of the fields called Spatial/SpatioTemporal Databases, Moving Objects Databases (MOD) and Geographic Information Systems (GIS) [2, 5, 11]. To give an intuitive idea about the magnitude - according to Mc Kinsey survey from 2011 [9], the volume of locationin-time data exceeds the order of Peta-Bytes per year just from smartphones - and this is only the "pure" GPS (Global Positioning System) data. Including the cell-towers location data would boost the size by two orders of magnitude - however, this is not even close to the full magnitude of the variety of location-related data contained in numerous tweets and other social networks based communications (which is of interest for applications such as behavioral marketing).

The location of a given moving object at a particular time instant is often obtained by some GPS based device [15]. Such sensed location data may be further combined with data from different board sensing devices on board the moving entities - e.g., U.S. Xpress gathers 900 to 970 data elements of various engine/component readings [7].

Permission to make digital or hard copies of all or part of this work for personal or classroom use is granted without fee provided that copies are not made or distributed for profit or commercial advantage and that copies bear this notice and the full citation on the first page. Copyrights for components of this work owned by others than ACM must be honored. Abstracting with credit is permitted. To copy otherwise, or republish, to post on servers or to redistribute to lists, requires prior specific permission and/or a fee. Request permissions from Permissions@acm.org.

MobiGIS'15, November 03-06, 2015 Bellevue, WA, USA

Copyright 2015 ACM ISBN 978-1-4503-3977-3/15/11

DOI: http://dx.doi.org/10.1145/2834126.2834818 .
However, since the 1920s when the traffic signals were still manually controlled, several generations of sensor types have been developed and deployed along road segments in various states, all for the purpose of more efficient traffic management [14]. The types of such sensors vary from the older pressure-sensitive ones introduced in 1931, to more modern laser-based sensors sensors and quite a few different types have been commercialized and used in day-to-day practical settings. For example, the AMR sensor developed by Honeywell is a type of magnetic sensor with low cost. The WiEye is a passive infrared sensor that can be installed on top of motes to sense road condition. The variation of sensing technologies may affect the manner of how a motion is modeled, in order to capitalize on the capabilities of a particular type of sensor and/or combination of their readings [1,4].

Regardless of how location data is obtained, there is the inevitable component of uncertainty due to:

- Imperfection of the localization devices (e.g., even differential GPS has errors within the range of a few meters).

- The measurements performed in discrete time-instants are used to model a continuous motion.

Thus, the best one can hope for is to incorporate some type of a "boundary" of the uncertainty, at various stages of the data management - from linguistic constructs for posing the queries of interest, to processing algorithms $[6,12,13]$. The main motivation for this talk is based on the observation that the state of the art has a lot of room for improvement in terms of models and algorithmic approaches that would combine (i.e., fuse) uncertain location data from different sources. Specifically, we postulate that a proper consideration of the joint impact of the uncertainties from multiple sources can tighten the boundaries of the objects whereabouts.

\section{TALK OUTLINE}

This talk will consist of three main portions:

1. The first part of the talk will give an overview of the problem and discuss the state of the art in the uncertainty management for moving objects. Subsequently, a brief overview of the data fusion will be presented (e.g., combining audio and video data has been a common activity for decades, complicated by the quest to compress the data for the purpose of more efficient 
transmission) [3]. The last portion of this part of the talk will be allocated for some preliminary background in the context of tracking/localization of moving objects.

2. In the second part of the talk, we will present a detailed discussion (from an actual case-study) on combining the uncertain location data from two sources: GPS and roadside sensors [17]. We will also present some preliminary experimental observations demonstrating the impact of such fusion in terms of reducing the number of false-positives in basic spatio-temporal queries.

3. The last part of the talk will discuss several contextbased extensions to the issues related to fusing uncertain location data from multiple sources. Specifically, we will discuss scenarios from geo-social networks and symbolic trajectories $[8,10,16]$, and will address some alternative measures of the benefits of fusing such data. The final portion of this part of the talk, in some sense, will attempt to present a vision about "the other side of fusing uncertain data" - e.g., how to improve existing privacy-related approaches from becoming weaker in the presence of multiple data sources; how to extract a sentiment from multiple data-feeds [18]; etc...

\section{SPEAKER BIO}

Goce Trajcevski is an Assistant Chairman with the Department of Electrical Engineering and Computer Science at Northwestern University. received his B.Sc. degree in Informatics and Automation from the University of Sts. Kiril i Metodij, and his MS and PhD degrees from the Department of Computer Science at the University of Illinois at Chicago. His broad research interests are in the areas of spatio-temoral data management, compression of mobile data, routing and data management in wireless sensor networks, and reactive behavior in dynamic systems. He has published over 90 papers in refereed conferences and journals, along with 4 book chapters and encyclopediae chapters. He has received the Best Paper Award at the 7th International Conference on Cooperative Information Systems (CoopIS, 2000), Best Paper Award at the 11th IEEE International Conference on Mobile Data Management (MDM, 2010) and Best Short Paper Award at the 16th ACM International Conference on Modeling, Analysis and Simulation of Wireless and Mobile Systems (MSWiM, 2013). His research has been funded by the National Science Foundation, Office of Naval Research, BEA Corp., Northrop Grumman Corp., and Walter P. Murphy Society. He has served as an associate editor for ACM DiSC, and is presently serving as an associate editor of GeoInformatica and ACM Transactions on Spatial Algorithms and Systems (TSAS) journals. He has also served as a General Co-Chair of ICDE 2014, PC Co-Chair of ADBIS 2014, and in various roles in the organizing committees of numerous conferences and workshops.

\section{REFERENCES}

[1] U. S. Department of Transportation. Travel monitoring and traffic volume, 2014. Office of Highway Policy Information.

[2] R. H. Güting and M. Schneider. Moving Objects Databases. Morgan Kaufmann, 2005.
[3] A. K. Katsaggelos, S. Bahaadini, and R. Molina. Audiovisual fusion: Challenges and new approaches. Proceedings of the IEEE, 103(9):1635-1653, 2015.

[4] B. Khaleghi, A. M. Khamis, F. Karray, and S. N. Razavi. Multisensor data fusion: A review of the state-of-the-art. Information Fusion, 14(1):28-44, 2013.

[5] M. Koubarakis, T. Sellis, A. Frank, S. Grumbach, R. Güting, C. Jensen, N. Lorentzos, Y. Manolopoulos, E. Nardelli, B. Pernici, H.-J. Scheck, M. Scholl, B. Theodoulidis, and N. Tryfona, editors. Spatio-Temporal Databases - the CHOROCHRONOS Approach. Springer-Verlag, 2003.

[6] B. Kuijpers and W. Othman. Trajectory databases: data models, uncertainty and complete query languages. Journal of Computer and System Sciences, 2009. doi:10.1016/j.jcss.2009.10.002.

[7] T. Leonard. Delivering deeper insights with big data and real-time analytics, 2012.

[8] G. Liu, R. Wang, J. Buckley, and H. M. Zhou. A wordnet-based semantic similarity measure enhanced by internet-based knowledge. In Proceedings of the 23rd International Conference on Software Engineering \& Knowledge Engineering (SEKE'2011), pages 175-178, 2011.

[9] Mckinsey Global Institute. Big data: The next frontier for innovation, competition, and productivity, 2011.

[10] C. Parent, S. Spaccapietra, C. Renso, G. L. Andrienko, N. V. Andrienko, V. Bogorny, M. L. Damiani, A. Gkoulalas-Divanis, J. A. F. de Macêdo, N. Pelekis, Y. Theodoridis, and Z. Yan. Semantic trajectories modeling and analysis. ACM Comput. Surv., 45(4):42, 2013.

[11] P. Rigaux, M. Scholl, and A. Voisard. Introduction to Spatial Databases: Applications to GIS. Morgan Kauffmann, 2000.

[12] G. Trajcevski, R. Tamassia, I. Cruz, P. Scheuermann, D. Hartglass, and C. Zamierowski. Ranking continuous nearest neighbors for uncertain trajectories. VLDB J., 20(5):767-791, 2011.

[13] G. Trajcevski, O. Wolfson, K. Hinrichs, and S. Chamberlain. Managing uncertainty in moving objects databases. ACM Trans. Database Syst., 29(3), 2004.

[14] Turner-Fairbank Highway Research Center. Traffic Detector Handbook. U.S. Department of transportation.

[15] United States Department of Defense. Navstar gps: Global positioning system standard, 2008.

[16] Z. Yan, N. Giatrakos, V. Katsikaros, N. Pelekis, and Y. Theodoridis. Setrastream: Semantic-aware trajectory construction over streaming movement data. In SSTD, pages 367-385, 2011.

[17] B. Zhang, G. Trajcevski, and L. Liu. Towards fusing uncertain location data from heterogeneous sources. GeoInformatica, 2015. (accepted, to appear).

[18] K. Zhang, Y. Xie, Y. Cheng, D. Honbo, D. Downey, A. Agrawal, W. Liao, and A. N. Choudhary. Sentiment identification by incorporating syntax, semantics and context information. In The 35th International ACM SIGIR conference, pages 1143-1144, 2012. 